\title{
HIGHER ORDER CONSERVATION LAWS
}

\author{
A. P. STONE
}

\section{Introduction}

Let $A$ denote the ring of germs of analytic functions at a point of an analytic manifold, and $\mathscr{E}$ the localization of the $A$-module of differential forms on this manifold. Let $\underline{h} \in \operatorname{Hom}_{A}(\mathscr{E}, \mathscr{E})$. Then $\theta \in \mathscr{E}$ is said to be a conservation law for $\underline{h}$ if both $\theta$ and $\underline{h} \theta$ are exact. More generally, $\theta$ is a conservation law for a finite collection of elements $\underline{h}_{i} \in \operatorname{Hom}(\mathscr{E}, \mathscr{E})$ if $\theta$ and $\underline{h}_{i} \theta$ are all exact. The problem which has been investigated in previous papers (see [4], [5], and [6]) has been that of determining all conservation laws $\theta \in \mathscr{E}$ for a given finite collection of endomorphisms $\underline{h}_{i}$ of $\mathscr{E}$. Certain conditions have usually been imposed on the $\underline{h}_{i}$, namely that the $\underline{h}_{i}$ have distinct eigenvalues and that certain concomitants associated with the $\underline{h}_{i}$ vanish identically.

The problem which is studied in this paper generalizes the conservation law problem in another direction. Let $\Lambda^{p} \mathscr{E}$ denote the differential forms of degree $p$, and $T \in \operatorname{Hom}_{A}\left(\Lambda^{p} \mathscr{E}, \Lambda^{p} \mathscr{E}\right)$. Then $\theta \in \Lambda^{p} \mathscr{E}$ is called a conservation law of order $p$ for $T$, if both $\theta$ and $T \theta$ are exact. The problem is then one of determining all conservation laws for a given $T$. In the sequel the case in which elements $T$ are induced by an element $\underline{h} \in \operatorname{Hom}_{A}(\mathscr{E}, \mathscr{E})$ is considered, and the main result is stated in Theorem 3.4. An alternate characterization of conservation laws is then given in $\S 4$.

\section{Preliminaries}

Let $\left(u^{1}, \cdots, u^{n}\right)$ form a local coordinate system in $A$ at some point $P$ of the manifold at which $u^{i}(P)=0$. If $d$ is the operation of exterior differentiation, then $\left(d u^{1}, \cdots, d u^{n}\right)$ is locally a basis for $\mathscr{E}$ which has dimension $n$. Moreover, $\mathscr{E}$ is a free $A$-module, and the exterior algebra $\Lambda^{*} \mathscr{E}$ generated by $\mathscr{E}$ is a direct sum

$$
\Lambda^{*} \mathscr{E}=\Lambda^{0} \mathscr{E} \oplus \Lambda^{1} \mathscr{E} \oplus \cdots \oplus \Lambda^{n} \mathscr{E},
$$

where $\Lambda^{0} \mathscr{E}=A$ and $\Lambda^{1} \mathscr{E}=\mathscr{E}$. The dimension of $\Lambda^{p} \mathscr{E}$, the space of differential

Communicated by A. Nijenhuis, September 19, 1968. This research was supported in part by NSF Grant GP-6560. 
forms of degree $p$, is $\left(\begin{array}{l}n \\ p\end{array}\right)$. If $\underline{h} \in \operatorname{Hom}(\mathscr{E}, \mathscr{E})$, then the endomorphism $h$, sometimes called a vector 1 -form, induces certain homomorphisms

$$
\Lambda^{p} \mathscr{E} \stackrel{h_{(q)}}{\longleftarrow} \Lambda^{p} \mathscr{E}, \quad 0 \leq q \leq p,
$$

of $\Lambda^{*} \mathscr{E}$, which are defined by setting

$$
\begin{aligned}
& h^{(q)}\left(\theta_{1} \wedge \cdots \wedge \theta_{p}\right) \\
& =\frac{1}{(p-q) ! q !} \sum_{\Pi}|\Pi| \cdot\left(\underline{h} \theta_{\Pi(1)} \wedge \cdots \wedge \underline{h} \theta_{\Pi(q)}\right) \wedge \theta_{\Pi(q+1)} \wedge \cdots \wedge \theta_{\Pi(p)}
\end{aligned}
$$

where $\theta_{i} \in \mathscr{E}, \Pi$ runs through all permutations of $(1,2, \cdots, p)$, the signature of the permutation $\Pi$ is denoted by $|\Pi|$, and the transformation $h^{(0)}$ is taken to be the identity on $\Lambda^{p} \mathscr{E}$.

The following result which is utilized in $\S 3$ asserts that $h^{(q)}$ can be expressed in terms of $h^{(1)}, h^{(2)}, \cdots, h^{(q-1)}$.

Lemma 2.1. Let $\theta \in \Lambda^{p} \mathscr{E}$. Then

$$
\begin{aligned}
{\left[\left(h^{q}\right)^{(1)}-\right.} & \left.\left(h^{q-1}\right)^{(1)} h^{(1)}+\cdots+(-1)^{q-1} h^{(1)} h^{(q-1)}\right] \theta \\
& =\left\{\begin{array}{l}
0, \quad q>p \\
(-1)^{q-1} q h^{(q)} \theta, \quad q \leq p .
\end{array}\right.
\end{aligned}
$$

Proof. Suppose first that $1<(j+1) \leq(q-1) \leq q \leq p$, and let $\theta \in \Lambda^{p} \mathscr{E}$ have the decomposition $\theta=\theta_{1} \wedge \cdots \wedge \theta_{p}$ where the $\theta_{i} \in \mathscr{E}$. If one considers any two consecutive terms in formula (2.2), then a careful analysis yields the following results. First, an expansion of $\left(h^{q-j}\right)^{(1)} h^{(j)}\left[\theta_{1} \wedge \cdots \wedge \theta_{p}\right]$ leads to $\left(\begin{array}{l}p \\ j\end{array}\right)\left(\begin{array}{l}p \\ 1\end{array}\right)$ terms of two types. There are $\left(\begin{array}{l}p \\ j\end{array}\right)\left(\begin{array}{l}j \\ 1\end{array}\right)$ distinct terms of the form

$$
\underline{h}^{q-j+1} \theta_{i_{1}} \wedge \underline{h} \theta_{i_{2}} \wedge \cdots \wedge \underline{h} \theta_{i_{j}} \wedge \theta_{i_{j+1}} \wedge \cdots \wedge \theta_{i_{p}}
$$

and there are also $\left(\begin{array}{l}p \\ j\end{array}\right)\left(\begin{array}{c}p-j \\ 1\end{array}\right)$ distinct terms of the form

$$
\underline{h} \theta_{i_{1}} \wedge \cdots \wedge \underline{h} \theta_{i_{j}} \wedge \underline{h}^{q-j} \theta_{i_{j+1}} \wedge \theta_{i_{j+2}} \wedge \cdots \wedge \theta_{i p}
$$

Similarly, $\left(h^{q-j-1}\right)^{(1)} h^{(j+1)}\left[\theta_{1} \wedge \cdots \wedge \theta_{p}\right]$ yields $\left(\begin{array}{c}p \\ j+1\end{array}\right)\left(\begin{array}{l}p \\ 1\end{array}\right)$ terms, of which $\left(\begin{array}{c}p \\ j+1\end{array}\right)\left(\begin{array}{c}j+1 \\ 1\end{array}\right)$ are of the form

$$
\underline{h} \theta_{i_{1}} \wedge \cdots \wedge \underline{h} \theta_{i_{j}} \wedge \underline{h}^{q-j} \theta_{i_{j+1}} \wedge \theta_{i_{j+2}} \wedge \cdots \wedge \theta_{i_{p}}
$$

while $\left(\begin{array}{c}p \\ j+1\end{array}\right)\left(\begin{array}{c}p-j-1 \\ 1\end{array}\right)$ terms are of the form 


$$
\underline{h} \theta_{i_{1}} \wedge \cdots \wedge \underline{h} \theta_{i_{j+1}} \wedge \underline{h}^{q-j-1} \theta_{i_{j+2}} \wedge \theta_{i_{j+3}} \wedge \cdots \wedge \theta_{i_{p}} .
$$

Since $\left(\begin{array}{l}p \\ j\end{array}\right)\left(\begin{array}{c}p-j \\ 1\end{array}\right)-\left(\begin{array}{c}p \\ j+1\end{array}\right)\left(\begin{array}{c}j+1 \\ 1\end{array}\right)=0$ and $\left(\begin{array}{c}p \\ j+1\end{array}\right)\left(\begin{array}{c}p-j-1 \\ 1\end{array}\right)=q\left(\begin{array}{l}p \\ q\end{array}\right)$ when $j+1=q-1$, formula (2.2) is established in the case where $q \leq p$. If one uses the convention that $h^{(j)} \theta=0$ whenever $j>p$, then a slight modification in the preceding argument will establish formula (2.2) when $q>p$.

Let $E=\operatorname{Hom}(\mathscr{E}, A)$ denote the dual $A$-module of vector fields. If $L \in E$ and $\theta \in \mathscr{E}$, then an endomorphism $\underline{h}$ of $\mathscr{E}$ is distinguished from its adjoint by writing $\underline{h} \theta$ when the action of $\underline{h}$ is on $\theta$, or $L \underline{h}$ when $\underline{h}$ acts on $L$; that is, $\langle L \underline{h}, \theta\rangle=\langle L, \underline{h} \theta\rangle \in A$. The differential concomitant $[\underline{h}, \underline{k}]$ of endomorphisms $\underline{h}$ and $\underline{k}$ is an element of $\operatorname{Hom}_{A}(\mathscr{E}, \mathscr{E} \wedge \mathscr{E})$ and is defined by setting

$$
\begin{aligned}
{[\underline{h}, \underline{k}] \theta=\frac{1}{2}\{} & -\left[h^{(1)} k^{(1)}-(h k)^{(1)}\right] d \theta \\
& \left.+\left[h^{(1)} d \underline{k} \theta+k^{(1)} d \underline{h} \theta\right]-[d \underline{h k} \theta+d \underline{k h} \theta]\right\}
\end{aligned}
$$

for any $\theta \in \mathscr{E}$. A dual characterization of $[\underline{h}, \underline{k}]$ as an element of $\operatorname{Hom}_{\mathbb{A}}(E \wedge E, E)$ may also be obtained. For any $L$ and $M \in E$ it is given as in [1] by the formula

$$
\begin{aligned}
(L \wedge M)[\underline{h}, \underline{k}]= & \frac{1}{2}\{[L, M] \underline{h} \underline{k}+[L, M] \underline{k} \underline{h}+[L \underline{h}, M \underline{k}]+[L \underline{k}, M \underline{h}] \\
& -[L \underline{h}, M] \underline{k}-[L \underline{k}, M] \underline{h}-[L, M \underline{h}] \underline{k}-[L, M \underline{k}] \underline{h}\} .
\end{aligned}
$$

The special case $\underline{k}=\underline{h}$ yields the Nijenhuis tensor $[\underline{h}, \underline{h}]$ of $\underline{h}$; in this case the preceding formulas reduce to

$$
[\underline{h}, \underline{h}] \theta=-h^{(2)} d \theta+h^{(1)} d \underline{h} \theta-d \underline{h}^{2} \theta, \quad \theta \in \mathscr{E},
$$

and

$$
(L \wedge M)[\underline{h}, \underline{h}]=[L, M] \underline{h}^{2}+[L \underline{h}, M \underline{h}]-[L \underline{h}, M] \underline{h}-[L, M \underline{h}] \underline{h},
$$

where $L$ and $M \in E$.

If $L_{*} \underline{h}$ denotes Lie differentiation of $\underline{h}$ with respect to a vector field $L$, then equations (2.4) and (2.6) may be rewritten in the form

$$
(L \wedge M)[\underline{h}, \underline{k}]=\frac{1}{2} M\left\{L \underline{h}_{*} \underline{k}+L \underline{k}_{*} \underline{h}-\left(L_{*} \underline{h}\right) \underline{k}-\left(L_{*} \underline{k}\right) \underline{h}\right\},
$$

and

$$
(L \wedge M)[\underline{h}, \underline{h}]=M\left\{L \underline{h}_{*} \underline{h}-\left(L_{*} \underline{h}\right) \underline{h}\right\}
$$


Consequently one may use (2.8) to conclude that $[\underline{h}, \underline{h}]=0$ if and only if $L \underline{h}^{i}{ }^{i} \underline{h}^{j}=\left(L_{*} \underline{h}^{j}\right) \underline{h}^{i}$ for any $L \in E$ and any pair of nonnegative integers $(i, j)$. Hence from (2.7) one can obtain the result as in [2] that the vanishing of $[\underline{h}, \underline{h}]$ implies the vanishing of $\left[\underline{h}^{i}, \underline{h}^{j}\right]$. Since this last statement is needed in the sequel, it is stated formally as a lemma.

Lemma 2.2. If $[\underline{h}, \underline{h}]=0$, then $\left[\underline{h}^{i}, \underline{h}^{j}\right]=0$ for any pair of nonnegative integers $(i, j)$.

The following result is a consequence of formula (5.9) in [1], and Lemma 2.2.

Lemma 2.3. If $[\underline{h}, \underline{h}]=0$ and $\theta \in \Lambda^{p} \mathscr{E}$, then

$$
\begin{aligned}
& {\left[\left(h^{i+j}\right)^{(1)}-\left(h^{i}\right)^{(1)}\left(h^{j}\right)^{(1)}\right] d \theta} \\
& +\left[\left(h^{i}\right)^{(1)} d\left(h^{j}\right)^{(1)} \theta+\left(h^{j}\right)^{(1)} d\left(h^{i}\right)^{(1)} \theta\right] \\
& -\left[d\left(h^{i+j}\right)^{(1)} \theta+d\left(h^{i}\right)^{(1)}\left(h^{j}\right)^{(1)} \theta\right]=0,
\end{aligned}
$$

where $i$ and $j$ are any positive integers.

Lemmas 2.1 and 2.3 may then be combined to yield the following corollary.

Corollary 2.4. If $[\underline{h}, \underline{h}]=0$ and $\theta \in \Lambda^{p} \mathscr{E}$, then

$$
-h^{(2)} d \theta+h^{(1)} d h^{(1)} \theta-d\left[\left(h^{2}\right)^{(1)}+h^{(2)}\right] \theta=0 .
$$

Note that if $p=1$, then equation (2.10) reduces to equation (2.5).

\section{Induced transformations}

In this section the special case of the problem announced in $\S 1$ is treated. It is assumed that $\theta \in \Lambda^{p} \mathscr{E}, 1 \leq p \leq n$, and that $\underline{h} \in \operatorname{Hom}(\mathscr{E}, \mathscr{E})$ is given, has distinct eigenvalues $\lambda_{1}, \cdots, \lambda_{n}$ and satisfies $[\underline{h}, \underline{h}]=0$. An element $\theta \in \Lambda^{p} \mathscr{E}$ is called a conservation law of order $p$ for $h^{(q)}, 0 \leq q \leq p \leq n$, if and only if $\theta$ and $h^{(q)} \theta$ are (locally) exact forms.

It should be observed that in the case $p=1$ (i.e., $\theta$ is a differential form of degree 1) the vanishing of $[\underline{h}, \underline{h}]$ implies $\theta$ is a conservation law for $\underline{h}^{q}, q \geq 2$, whenever $\theta$ is a conservation law for $\underline{h}$. However when $\theta$ is a $p$-form, with $p>1$, the condition $[\underline{h}, \underline{h}]=0$ does not imply an analogous result. That is, if $\theta$ is a conservation law for $h^{(1)}$, then $\theta$ need not be a conservation law for either $\left(h^{q}\right)^{(1)}$ or $h^{(q)}$ when $q \geq 2$. The following example will illustrate this remark. It is assumed that Lemma 3.1 is already at our disposal.

Let $n=3$ and $p=2$, and assume that $\underline{h}$ has the distinct eigenvalues $\lambda_{1}, \lambda_{2}$, and $\lambda_{3}$ and satisfies $[\underline{h}, \underline{h}]=0$. Lemma 3.1 then guarantees the existence of a basis of exact eigenforms $d u^{1} d u^{2}$, and $d u^{3}$. Consequently, if $\theta \in \mathscr{E} \wedge \mathscr{E}$ has the form

$$
\theta=\left(\lambda_{1}-\lambda_{2}\right) u_{3} d u^{1} \wedge d u^{2}+\left(\lambda_{1}-\lambda_{3}\right) u_{2} d u^{1} \wedge d u^{3}+\left(\lambda_{2}-\lambda_{3}\right) u_{1} d u^{2} \wedge d u^{3}
$$


then $d \theta=d h^{(1)} \theta=0$ and $\theta$ is a conservation law for $h^{(1)}$. However

$$
d\left(h^{2}\right)^{(1)} \theta=\gamma\left(d u^{1} \wedge d u^{2} \wedge d u^{3}\right)
$$

and

$$
d h^{(2)} \theta=-\gamma\left(d u^{1} \wedge d u^{2} \wedge d u^{3}\right)
$$

where $\gamma$ is the Vandermonde determinant

$$
r=\left|\begin{array}{lll}
1 & 1 & 1 \\
\lambda_{1} & \lambda_{2} & \lambda_{3} \\
\lambda_{1}^{2} & \lambda_{2}^{2} & \lambda_{3}^{2}
\end{array}\right| \neq 0,
$$

and consequently $\theta$ can not be a conservation law for $\left(h^{2}\right)^{(1)}$ or $h^{(2)}$.

Lemma 3.1. If $h$ has distinct eigenvalues and satisfies $[\underline{h}, \underline{h}]=0$, then there exist coordinates $v^{1}, \cdots, v^{n}$ such that $\partial / \partial v^{i}$ are eigenvector field of $h$ and the corresponding eigenvalues $\lambda_{i}$ are functions of $v^{i}$ alone.

The proof of this lemma is omitted as it appears elsewhere (see [3]). As a consequence of this lemma, $\mathscr{E}$ has an eigenvector basis consisting of exact forms $\left(d v^{1}, \cdots, d v^{n}\right)$, and hence the $\left(\begin{array}{l}n \\ p\end{array}\right)$ elements $d v^{i_{1}} \wedge \cdots \wedge d v^{i}$ form a basis for $\Lambda^{p} \mathscr{E}$.

Lemma 3.2. If $h$ has distinct eigenvalues and satisfies $[\underline{h}, \underline{h}]=0$, and $\theta \in \Lambda^{p} \mathscr{E}$ is a conservation law for $h^{(1)}, h^{(2)}, \cdots, h^{(q)}$ with $q \leq p$, then $\theta$ is also a conservation law for $\left(h^{q-j}\right)^{(1)} h^{(j)}$ and $h^{(q-j)} h^{(j)}$ where $0 \leq j \leq q$.

Proof. It may be assumed that $p \geq 2$. Hence if $q=2$ and $\theta \in \Lambda^{p} \mathscr{E}$, then equations (2.2) and (2.10) yield respectively

$$
\begin{aligned}
\left(h^{2}\right)^{(1)}-h^{(1)} h^{(1)} & =-2 h^{(2)}, \\
d\left[\left(h^{2}\right)^{(1)}+h^{(2)}\right] \theta & =0,
\end{aligned}
$$

and hence $\theta$ is a conservation law for $\left(h^{2}\right)^{(1)}$ and $h^{(1)} h^{(1)}$ whenever $\theta$ is a conservation law for $h^{(1)}$ and $h^{(2)}$. Next let $q=p$, and suppose that $\theta$ is a conservation law for $h^{(1)}, h^{(2)}, \cdots, h^{(p)}$, and also for $\left(h^{p-j-1}\right)^{(1)} h^{(j)}$ and $h^{(p-j-1)} h^{(j)}$ with $0 \leq j \leq(p-1)$. If $i=1$ is fixed and $j=1,2, \cdots,(p-1)$ in equation (2.9), then the following set of $(p-1)$ equations is obtained:

$$
\begin{aligned}
d\left[\left(h^{p}\right)^{(1)}+h^{(1)}\left(h^{p-1}\right)^{(1)}\right] \theta & =0, \\
d\left[\left(h^{p-1}\right)^{(1)}+h^{(1)}\left(h^{p-2}\right)^{(1)}\right] h^{(1)} \theta & =0, \\
d\left[\left(h^{p-2}\right)^{(1)}+h^{(1)}\left(h^{p-3}\right)^{(1)}\right] h^{(2)} \theta & =0, \\
\cdot \cdot \cdot \cdot \cdot \cdot \cdot \cdot & \cdot \\
d\left[\left(h^{2}\right)^{(1)}+h^{(1)} h^{(1)}\right] h^{(p-2)} \theta & =0 .
\end{aligned}
$$


If these equations are combined and formula (2.2) is applied, one then obtains

$$
(-1)^{p} p d\left[h^{(1)} h^{(p-1)}\right] \theta=0,
$$

and hence $\theta$ is a conservation law for $h^{(1)} h^{(p-1)}$. Slight modifications in the preceding argument then serve to establish this lemma.

Corollary 3.3. Let $h$ have distinct eigenvalues and vanishing Nijenhuis torsion $[h, h]$. If $\theta \in \Lambda^{p} \mathscr{E}$ is a conservation law for $h^{(1)}, h^{(2)}, \cdots, h^{(p)}$, then $\theta$ is also a conservation law for $\left(h^{p}\right)^{(1)}$.

The preceding lemmas can now be used to obtain the following main result of this paper, which gives a local representation of any $p$-th order conservation law.

Theorem 3.4. If $\underline{h} \in \operatorname{Hom}(\mathscr{E}, \mathscr{E})$ has distinct eigenvalues $\lambda_{1}, \cdots, \lambda_{n}$ and vanishing Nijenhuis torsion $[\underline{h}, \underline{h}]$, then $\theta \in \Lambda^{p} \mathscr{E}$ is a conservation law for $h^{(1)}, \cdots, h^{(p)}$ if and only if

$$
\theta=\sum_{i_{1}<\cdots<i_{p}} f_{i_{1} \cdots i_{p}}\left(v^{i_{1}}, \cdots, v^{i_{p}}\right) d v^{i_{1}} \wedge \cdots \wedge d v^{i_{p}},
$$

where $\left\{d v^{1}, \ldots, d v^{n}\right\}$ is an eigenform basis for $\mathscr{E}$.

Proof. Suppose that $\theta$ has the form (3.1) and let $\partial f_{i_{1} \ldots i_{p}} / \partial v^{j} \equiv f_{i_{1} \ldots i_{p, j}}$ for convenience of notation. Then $d \theta=0$ since $f_{i_{1} \ldots i_{p}, j}=0$ for $j \neq i_{1}, \cdots, i_{p}$. Similarly since $\lambda_{i}=\lambda_{i}\left(v^{i}\right)$ it is also clear that $d h^{(i)} \theta=0$, and hence $\theta$ is a conservation law for $h^{(i)}$ with $0 \leq i \leq p$.

Conversely if $\theta$ is a conservation law for $h^{(i)}$, then $d h^{(i)} \theta=0$ and hence $d\left(h^{i}\right)^{(1)} \theta=0$ also. Now suppose that $\theta$ has the form

$$
\theta=\sum_{i_{1}<\cdots<i_{p}} f_{i_{1} \cdots i_{p}}\left(v^{1}, \cdots, v^{n}\right) d v^{i_{1}} \wedge \cdots \wedge d v^{i_{p}} .
$$

In the case that $p=n$, every element $\theta \in \Lambda^{n} \mathscr{E}$ is a conservation law for $h^{(i)}$, $0 \leq i \leq n$, and hence only the cases where $0<p<n$ will be considered. If $\theta \in \Lambda^{p} \mathscr{E}$, then the conditions $d\left(h^{i}\right)^{(1)} \theta=0,0 \leq i \leq p$, lead to $\left(\begin{array}{c}n \\ p+1\end{array}\right)$ systems of homogeneous partial differential equations, since the number of systems must be the same as the dimension of $\Lambda^{p+1} \mathscr{E}$. Each system must contain $(p+1)$ equations in $(p+1)$ unknowns. Let us now examine a particular system in detail. If $\theta$ is of the form (3.2), then

$$
d \theta=\sum_{j_{1}<\cdots<j_{p+1}}\left\{(-1)^{p} \frac{1}{p !} \sum_{I I}|\Pi| \cdot F_{j_{1} \ldots j_{p}, j_{p+1}}\right\} d v^{j_{1}} \wedge \cdots \wedge d v^{j_{p+1}},
$$

where $F_{j_{1} \cdots j_{p}, j_{p+1}} \equiv f_{\Pi\left(j_{1}\right) \cdots n\left(j_{p}\right), \pi\left(j_{p+1}\right)},\left(j_{1}, \cdots, j_{p_{+1}}\right)$ is contained in $(1,2$, $\cdots, n)$, and the signature of the permutation $\Pi$ of $\left(j_{1}, \cdots, j_{p_{+1}}\right)$ is denoted by $|\Pi|$. Note that if we set $f_{\Pi\left(j_{1}\right) \cdots \Pi_{(j p), \Pi\left(j_{p+1}\right)}}$ equal to $|\Pi| \cdot f_{\beta\left(j_{1}\right) \cdots \beta\left(j_{p}\right), \Pi\left(j_{p+1}\right)}$ where $\beta$ is any rearrangement of $\left(j_{1}, \cdots, j_{p}\right)$, then the coefficient of 
$d v^{j_{1}} \wedge \cdots \wedge d v^{j_{p+1}}$ which is a sum of $(p+1)$ ! terms can be reduced to a sum of $(p+1)$ terms. If the homomorphism $h^{(1)}$ defined by equation (2.1) is applied to $\theta$, then

$$
\begin{array}{r}
d h^{(1)} \theta=\sum_{j_{1}<\cdots<j_{p+1}}\left\{(-1)^{p} \frac{1}{p !} \sum_{n}\left(\lambda_{j_{1}}+\cdots+\lambda_{j_{p+1}}\right)|\Pi| \cdot F_{j_{1} \ldots j_{p}, j_{p+1}}\right\} \\
\cdot d v^{j_{1}} \wedge \cdots \wedge d v^{j_{p+1}}
\end{array}
$$

is a consequence of Lemma 3.1. Similarly, the computation of $d\left(h^{p}\right)^{(1)} \theta$ yields a result analogous to equation (3.4) in which the sum $\left(\lambda_{j_{1}}+\cdots+\lambda_{j_{p+1}}\right)$ is replaced by the sum $\left(\lambda_{j_{1}}^{p}+\cdots+\lambda_{j_{p+1}}^{p}\right)$.

For example, if $n=4$ and $p=2$, then the conditions $d\left(h^{i}\right)^{(1)} \theta=0,0 \leq i$ $\leq 2$, lead to four systems of equations and each system contains three equations in three unknowns. The equations of one such system can be written out explicitly as

$$
\begin{aligned}
f_{12,3}-f_{13,2}+f_{23,1} & =0, \\
\left(\lambda_{1}+\lambda_{2}\right) f_{12,3}-\left(\lambda_{1}+\lambda_{3}\right) f_{13,2}+\left(\lambda_{2}+\lambda_{3}\right) f_{23,1} & =0, \\
\left(\lambda_{1}^{2}+\lambda_{2}^{2}\right) f_{12,3}-\left(\lambda_{1}^{2}+\lambda_{3}^{2}\right) f_{13,2}+\left(\lambda_{2}^{2}+\lambda_{3}^{2}\right) f_{23,1} & =0,
\end{aligned}
$$

and this system has the unique solution $f_{12,3}=f_{13,2}=f_{23,1}=0$ since the eigenvalues are distinct. An examination of the other systems leads to the conclusion that $f_{i j, k}=0$ for any three distinct integers $i, j$, and $k$ which range over the set $(1,2,3,4)$ and consequently $f_{i j}=f_{i j}\left(v^{i}, v^{j}\right)$.

Thus the conditions $d\left(h^{i}\right)^{(1)}=0$ lead to a typical system of $(p+1)$ equations of the form

$$
\begin{aligned}
0= & \left(\lambda_{k_{1}}^{j}+\cdots+\lambda_{k_{p}}^{j}\right) f_{k_{1} \cdots k_{p}, k_{p+1}} \\
& -\cdots+(-1)^{p}\left(\lambda_{k_{2}}^{j}+\cdots+\lambda_{k_{p+1}}^{j}\right) f_{k_{2} \cdots k_{p+1}, k_{1}},
\end{aligned}
$$

where $0 \leq j \leq p$. For a fixed positive integer $p$ there are $\left(\begin{array}{c}n \\ p+1\end{array}\right)$ such systems. The sytem (3.5) has the unique solution $f_{k_{1} \ldots k_{p, k_{p+1}}}=0$ for all permutations of the set $\left(k_{1}, \cdots, k_{p+1}\right)$ since the determinant in Figure 1, which reduces to the Vandermonde determinant shown in Figure 2, is nonvanishing. An analysis of the remaining systems then leads to the general conclusion that $f_{j_{1} \ldots j_{p}, j_{p+1}}$ $=0$ for any $(p+1)$ distinct integers $\left(j_{1}, \ldots, j_{p}, j_{p+1}\right)$ belonging to the set $(1,2, \cdots, n)$. Consequently, the result that $f_{i_{1} \ldots i_{p}}=f_{i_{1} \ldots i_{p}}\left(v^{i_{1}}, \cdots, v^{i_{p}}\right)$ is obtained, and the proof of Theorem 3.4 is completed.

$$
\left|\begin{array}{ccc}
1 & \cdots & 1 \\
\lambda_{k_{1}}+\cdots+\lambda_{k_{p}} & \cdots & \lambda_{k_{2}}+\cdots+\lambda_{k_{p+1}} \\
\vdots & & \vdots \\
\lambda_{k_{1}}^{p}+\cdots+\lambda_{k_{p}}^{p} & \cdots & \lambda_{k_{2}}^{p}+\cdots+\lambda_{k_{p+1}}^{p}
\end{array}\right|
$$

Figure 1 


$$
\left|\begin{array}{cccc}
1 & \cdots & 1 & 1 \\
\lambda_{k_{1}} & \cdots & \lambda_{k_{p}} & \lambda_{k_{p+1}} \\
\lambda_{k_{1}}^{2} & \cdots & \lambda_{k_{p}}^{2} & \lambda_{k_{p+1}}^{2} \\
\vdots & & \vdots & \vdots \\
\lambda_{k_{1}}^{p} & \cdots & \lambda_{k_{p}}^{p} & \lambda_{k_{p+1}}^{p}
\end{array}\right|
$$

Figure 2

\section{An alternate characterization of conservation laws}

If one considers the set of equations

$$
h^{(j)} d \theta=d h^{(j)} \theta, \quad \theta \in \Lambda^{p} \mathscr{E}, \quad 1 \leq j \leq p,
$$

then it is clear that any $\theta$ which is a conservation law for $h^{(j)}$ satisfies this system. A similar remark can be made for the system $\left(h^{j}\right)^{(1)} d \theta=d\left(h^{j}\right)^{(1)} \theta$. Conversely, if $\theta$ satisfies the equations $\left(h^{j}\right)^{(1)} d \theta=d\left(h^{j}\right)^{(1)} \theta, 1 \leq j \leq 2$, and $\underline{h}$ has distinct eigenvalues and vanishing Nijenhuis tensor, then one may show that $\theta$ is a conservation law for $h^{(j)}$ and $\left(h^{j}\right)^{(1)}$ where $j$ is any positive integer. In the notation of [1] this last assertion is equivalent to the statement that conservation laws are solutions of the equations $d_{h} \theta=d_{h^{2}} \theta=0$.

Theorem 4.1. Let $[h, h]=0$. If $\theta \in \Lambda^{p} \mathscr{E}$ and satisfies the equations $\left(h^{j}\right)^{(1)} d \theta=d\left(h^{j}\right)^{(1)} \theta, 1 \leq j \leq 2$, then $\left(h^{j}\right)^{(1)} d \theta=d\left(h^{j}\right)^{(1)} \theta$ and $h^{(j)} d \theta=d h^{(j)} \theta$ for $2 \leq j \leq p$, and $h^{(j)} d \theta=0$ for $j>p$.

Proof. Since $[\underline{h}, \underline{h}]=0$, equation $(2.10)$ yields:

$$
-2 h^{(2)} d \theta+2 h^{(1)} d h^{(1)} \theta-2 d\left[\left(h^{2}\right)^{(1)}+h^{(2)}\right] \theta=0 .
$$

Equation (2.2) then implies

$$
-2 h^{(2)} d \theta+2 h^{(1)} d h^{(1)} \theta-2 d\left[h^{(1)} h^{(1)}-h^{(2)}\right] \theta=0,
$$

and hence $h^{(2)} d \theta=d h^{(2)} \theta$. Note that if $p=1$, then $h^{(2)} d \theta=0$.

Now suppose that $\left(h^{j}\right)^{(1)} d \theta=d\left(h^{j}\right)^{(1)} \theta$ and $h^{(j)} d \theta=d h^{(j)} \theta, 1 \leq j \leq(p-1)$; then equation (2.9) implies

$$
\begin{aligned}
0= & {\left[\left(h^{j+1}\right)^{(1)}-\left(h^{j}\right)^{(1)} h^{(1)}\right] d \theta } \\
& +\left[\left(h^{j}\right)^{(1)} d h^{(1)}+h^{(1)} d\left(h^{j}\right)^{(1)}\right] \theta \\
& -\left[d\left(h^{j+1}\right)^{(1)}+d h^{(1)}\left(h^{j}\right)^{(1)}\right] \theta,
\end{aligned}
$$

and consequently $\left(h^{j+1}\right)^{(1)} d \theta=d\left(h^{j+1}\right)^{(1)} \theta$. Since Lemma 2.1 yields

$$
\left(h^{j+1}\right)^{(1)}-\left(h^{j}\right)^{(1)} h^{(1)}+\cdots+(-1)^{j} h^{(1)} h^{(j)}=(-1)^{j}(j+1) h^{(j+1)},
$$


one thus obtains $h^{(j+1)} d \theta=d h^{(j+1)} \theta$ when $(j+1) \leq p$, and $h^{(j+1)} d \theta=0$ when $(j+1)>p$.

Corollary 4.2. Suppose $h$ has distinct non-zero eigenvalues and satisfies $[\underline{h}, \underline{h}]=0$. If $\left(h^{j}\right)^{(1)} d \theta=d\left(h^{j}\right)^{(1)} \theta, 1 \leq j \leq 2$, for some $\theta \in \Lambda^{p} \mathscr{E}$, then $\theta$ is a conservation law for $h^{(j)}$ and $\left(h^{j}\right)^{(1)}$ for all positive integers $j$.

Proof. Theorem 4.1 yields two systems of equations, namely,

$$
\begin{aligned}
h^{(1)} d \theta & =d h^{(1)} \theta, \\
h^{(2)} d \theta & =d h^{(2)} \theta, \\
\vdots & \vdots \\
h^{(p)} d \theta & =d h^{(p)} \theta, \\
h^{(p+1)} d \theta & =0,
\end{aligned}
$$

and

$$
\begin{array}{cc}
h^{(1)} d \theta=d h^{(1)} \theta, \\
\left(h^{2}\right)^{(1)} d \theta=d\left(h^{2}\right)^{(1)} \theta, \\
\vdots & \vdots \\
\left(h^{p}\right)^{(1)} d \theta=d\left(h^{p}\right)^{(1)} \theta, \\
h^{(p+1)} d \theta=0 .
\end{array}
$$

Since the homomorphism $h^{(p+1)}$ acts on $(p+1)$-forms, it is invertible if and only if $\underline{h}$ is invertible. Hence $d \theta=0$ and the corollary is established.

It should be remarked that if $\underline{h}$ is singular then a nonsingular endomorphism $\underline{h}^{*}$ such that $\left[\underline{h}^{*}, \underline{h}^{*}\right]=[\underline{h}, \underline{h}]$ can be obtained simply by setting $\underline{h}^{*}=\underline{h}+\alpha I$ for some suitable choice of a constant $\alpha$.

\section{Conclusion}

The main import of Theorem 3.4 is the following observation. Given a conservation law $\theta$ of order $p$, equation (3.1) asserts that if $p$ is large then the conditions are less restrictive on the form of the coefficients which occur in the expression for $\theta$ in terms of the basis for $\Lambda^{p} \mathscr{E}$. Thus in the case $p=n$ everything in sight is a conservation law for $h^{(i)}$. If $p=n-1$, then $\theta$ is a conservation law for $h^{(i)}$ if and only if

$$
\theta=f_{12 \cdots(n-2)} d v^{1} \wedge \cdots \wedge d v^{(n-2)}+\cdots+f_{23 \cdots(n-1)} d v^{2} \wedge \cdots \wedge d v^{(n-1)},
$$

and the coefficient $f_{12 \ldots \hat{i} \ldots(n-1)}$ is a function of everything but $v^{i}$. On the other hand, for $p=1, \theta=f_{1} d v^{1}+\cdots+f_{n} d v^{n}$ is a conservation law for $\underline{h}$ if and only if each $f_{i}$ is a function of $v^{i}$ alone. 


\section{References}

[1] A. Frölicher \& A. Nijenhuis, Theory of vector-valued differential forms. I, Nederl. Akad. Wetensch. Proc. Ser. A, 59 (1956) 338-359.

[2] E. T. Kobayashi, A remark on the Nijenhuis tensor, Pacific J. Math. 12 (1962) 963-977.

[3] A. Nijenhuis, $X_{n-1}$-forming sets of eignevectors, Nederl. Akad. Wetensch. Proc. Ser. A, 54 (1951) 200-212.

[4] H. Osborn, Les lois de conservation, Ann. Inst. Fourier (Grenoble) 14 (1964) 7182.

[ 5 ] A. P. Stone, Analytic conservation laws, Ann. Inst. Fourier (Grenoble) 16 (1966) 319-327.

[6] - Generalized conservation laws, Proc. Amer. Math. Soc. 18 (1967) 868-873.

UNiversity OF Illinois, Chicago 